Clinical Expriance,

\title{
Clinical Analysis of Redo Aortic Root Replacement after Cardiac Surgery
}

\author{
Jianying Deng, ${ }^{1}$ Qianjin Zhong ${ }^{2}$ \\ ${ }^{1}$ Department of Cardiovascular Surgery, Chongqing Kanghua Zhonglian Cardiovascular Hospital, \\ Chong Qing 400015, China \\ ${ }^{2}$ Department of Cardiovascular Surgery, Army Medical Center of PLA, Chong Qing 400020, China \\ Email Address: 65673171@qq.com
}

\begin{abstract}
:
Objectives To explore the etologies, surgical methods and outcomes of redo aortic root replacement after cardiac surgery.

Methods Between February 2010 and February 2020, 41 patients who had at least one previous cardiac operation received aortic root replacement surgery in our hospita, including 27 males and 14 females with an average age of $49.5 \pm 10.2$ years. Indications for reoperation include: aortic sinus dilation and ascending aortic aneurysm in 20 cases $(48.8 \%)$, recurrent aortic dissection in 7 cases (17.0\%), aortic pseudoaneurysm in 4 cases $(9.8 \%)$, prosthetic valve endocarditis in 5 cases $(12.2 \%)$ and 5 cases $(12.2 \%)$ had paravalvular leakage. The redo operation was a midline thoracotomy, and Bentall operation was performed. In addition, 3 cases of mitral valve replacement surgery, 4 cases of mitral valvuloplasty, 8 cases of tricuspid valvuloplasty, 4 cases of coronary artery bypass graft surgery, 3 cases of Debakey I aortic dissection patients underwent Sun's surgery simultaneously.

Results The mean cardiopulmonary bypass time was 95 302 (168.1 \pm 41.2$) \mathrm{min}$, and the mean aortic crossclamp time was 45 135 $(84.5 \pm 22.3) \mathrm{min}$. Six patients $(14.6 \%)$ died during the postoperative hospitalization. The main causes of death were heart failure in 3 patients and septic shock in 3 patients. The follow-up time was 3 62 (30.3 \pm 15.5$)$ months. During the follow-up period, 3 cases died, including 1 case died of septic shock and 2 cases died of cerebral hemorrhage.

Conclusions Redo aortic root replacement is difficult to deal with, and the risk is high. Preoperative evaluation is required, appropriate surgical approach, adequate myocardial protection, and a complete surgical plan are essential to ensure the success of the operation.
\end{abstract}

Keywords: aortic root replacement; cardiac reoperation; aortic disease; surgery

\section{Introduction:}

Aortic root replacement surgery is the most effective way to treat aortic root aneurysm. In 1968, Bentall and De Bono ${ }^{1}$ first reported the use of a Teflon graft and Starr valve for aortic root replacement to treat aortic root diseases, opening a new era of aortic surgery.In some patients who needed redo caidiac surgery with normal aortic roots, there is no need to deal with the aortic roots. However, in some patients with abnormal aortic roots, such as aortic root aneurys, aortic root abscesses, severely calcified aortic roots, dilated aortic annulus, etc., Bentall surgery is a safe and reliable option. This article summarizes the preoperative, postoperative and follow-up results of patients undergoing redo Bentall surgery in our hospital after cardiac surgery.

\section{Materials And Methods:}

\subsection{Clinical data:}

From February 2010 to February 2020, 41 patients in our department underwent redo Bentall surgery, including 27 males and 14 females, aged 16-72 $(49.5 \pm 10.2)$ years old.In these cases, at least one heart operation had been performed in the past, and 6 of them had a history of two heart operations performed in the past.The interval between the two operations was 8 months to 25 years, with an 
average of $(7.0 \pm 7.5)$ years. 10 cases had a history of hypertension. The preoperative ECG showed atrial fibrillation in 6 cases. 4 cases underwent emergency surgery, of which 2 cases had prosthetic valve endocarditis (PVE) after aortic valve replacement, which caused acute left heart failure due to paravalvular leakage, and the other 2 cases recurred after aortic valve replacement emergency surgery for acute aortic dissection. Table 1 shows preoperative data of 41 patients.

The previous heart surgery included 4 cases of Bentall surgery (including 3 cases of total arch replacement + elephant stent implantation at the same time), 23 cases of aortic valve replacement surgery (including 2 cases of biological valves, and simultaneous mitral valve replacement or 15 cases of mitral valvuloplasty or tricuspid valvuloplasty, 2 cases of ascending aorta angioplasty, 2 cases of aortic root widening), 4 cases of aortic valvuloplasty (2 cases underwent type A preexcitation syndrome abnormal bypass conduction cut at the same time Surgery + ventricular septal defect repair + patent foramen ovale repair + mitral valvuloplasty), aortic root plasty + ascending aorta + total arch replacement + elephant trunk implantation + coronary artery bypass grafting (coronary artery bypass grafting, CABG) in 2 cases, ascending aorta + total arch replacement + elephant stent implantation in 2 cases, simple mitral valve replacement in 3 cases, radical resection of tetralogy of Fallot in 3 cases, and ventricular septal defect repair in 2 case. Table 1 shown this.

Etologies for reoperation included: aortic root dilation and ascending aortic aneurysm in 20 cases, recurrent aortic dissection in 7 cases (Debakey I

3 cases, Debakey II 4 cases), and 4 cases of aortic root pseudoaneurysm (both After Bentall, 2 cases had coronary anastomotic tear, 2 cases had valved catheter prosthesis and aortic root tear), 5 cases of PVE, 5 cases of paravalvular leak (4 cases of Behcet's disease, 1 case of Paravalvular leak closure failed). See Table 2.

\section{2 surgery methods:}

All 41 patients in this group used the original median sternal incision to enter the chest. During the establishment of cardiopulmonary bypass (CPB), only 2 cases were intubated through the ascending aorta, and the remaining 39 cases were intubated through the femoral artery. After thoracotomy, 4 patients underwent axillary artery or innominate artery cannulation at the same time. The femoral vein or the superior and inferior vena cava were selected for venous cannulation. CPB was established through right atrium cannulation in 4 patients. Our principle is to evaluate the distance between the sternum and the aorta and right ventricle through imaging examinations before surgery. When the distance is close or the right ventricular pressure is high, $\mathrm{CPB}$ is established through peripheral blood vessels first to avoid heart injury and hemorrhage during thoracotomy. The rest of the patients were opened after the establishment of CPB through the femoral vein. No serious bleeding and other complications occurred in the whole group of patients. In terms of myocardial protection, one patient received intermittent retrograde perfusion with cold-blooded myocardial protective solution for pulsating pseudoaneurysm of the aortic root, and the rest were intermittent antegrade perfusion. The lowest body temperature was $24.7-32.8(30.7 \pm 1.9){ }^{\circ} \mathrm{C}$. All patients underwent Bentall surgery again, and to avoid reoperation, mechanical valves were used. For coronary artery transplantation, if the adhesion around the coronary artery opening is severe and the dissection is difficult, and the coronary artery opening is not obvious, we used the intraluminal anastomosis method in 4 cases (13\%), and the rest were carefully dissected and separated out the button-shaped coronary artery opening. 1 patient underwent great saphenous vein anastomosis to lengthen the right coronary artery due to difficulty in right coronary artery anastomosis, 1 patient underwent a bypass from great saphenous vein to the proximal right coronary artery due to narrow right coronary artery opening, and at the same time closed right coronary artery Arterial opening. 3 cases of mitral valve replacement surgery, 4 cases of mitral valvuloplasty, 8 cases of tricuspid valvuloplasty, 4 cases of coronary artery bypass graft surgery, 3 cases of Debakey I aortic dissection patients underwent Sun's surgery at the same time .

\subsection{Ethical review:}

This study has been approved by the ethics committee of the Chongqing Kanghua Zhonglian Cardiovascular Hospital and Army Medical Center of PLA, and has been recognized as exempt from ethical review.

\subsection{Statistical analysis:}

The SPSS 22.0 software was used for statistical analysis. The measurement data was expressed as $\overline{\mathrm{x}} \pm \mathrm{s}$, and the counting data was expressed as a percentage. The comparison of means adopts t test. Using a two-sided test, $p<0.05$ was considered statistically significant. 
Table1.preoperative characteristics [cases $(\%) / \overline{\mathbf{x}} \pm \mathrm{s}]$

\begin{tabular}{|l|c|}
\hline Variable & Distribution \\
\hline Age & $49.5 \pm 10.2$ \\
\hline Gender, male & $27(65.9)$ \\
\hline BMI(kg/m ${ }^{2}$ & $21.5 \pm 4.2$ \\
\hline Hypertension & $10(24.4)$ \\
\hline diabetes & $4(9.8)$ \\
\hline Hyperlipidemia & $5(12.2)$ \\
\hline COPD & $2(4.9)$ \\
\hline Preoperative heart failure & $16(39.0)$ \\
\hline History of stroke & $2(4.9)$ \\
\hline Preoperative hemodialysis & $1(2.4)$ \\
\hline Peripheral vascular disease & $4(9.8)$ \\
\hline Coronary heart disease & $4(9.8)$ \\
\hline Atrial fibrillation & $6(14.6)$ \\
\hline Marfan syndrome & $2(4.9)$ \\
\hline Aortic dissection & $9(22.0)$ \\
\hline Heart function classification & \\
(NYHA) & \\
\hline I /II & $12(29.3)$ \\
\hline III/IV & $29(70.7)$ \\
\hline $\begin{array}{l}\text { Left ventricular ejection fraction } \\
\text { <50\% }\end{array}$ & $7(17.1)$ \\
\hline Emergency surgery & $4(9.8)$ \\
\hline Last operation method & \\
\hline Bentall surgery & $4(9.8)$ \\
\hline Aortic valve replacement & $23(56.1)$ \\
\hline Aortic valve/root formation & $4(9.8)$ \\
\hline Ascending aorta replacement & $2(4.9)$ \\
\hline Mitral valve replacement & $3(7.3)$ \\
\hline Tetralogy of Fallot repair & $3(7.3)$ \\
\hline Ventricular septal defect repair & $2(4.9)$ \\
\hline
\end{tabular}

Abbreviations: BMI, Body Mass Index; COPD: chronic obstructive pulmonary disease.

Note: Values are presented as $n(\%)$ or mean \pm standard deviation.

\section{Results:}

\subsection{Early clinical results:}

The CPB time of all patients was 95-302 $(168.1 \pm 41.2) \mathrm{min}$, and the aortic block time was 45-135 (84.5 \pm 22.3$) \quad$ min. During the hospitalization, 6 cases $(14.6 \%)$ died, 3 cases died of postoperative heart failure, 3 cases died of septic shock, mediastinal infection and lung infection. Four patients (9.8\%) underwent secondary thoracotomy for exploratory thoracotomy due to excessive pleural fluid in the early postoperative period. Postoperative complications included stroke in 2 cases $(4.9 \%)$, tracheotomy in 3 cases (7.3\%), postoperative renal failure in 5 cases $(12.2 \%)$ and poor incision healing in 3 cases (7.3\%). 7 cases $(17.1 \%)$ of postoperative ventilator ventilation time> $72 \mathrm{~h}$. The average ICU stay time was $(9.2 \pm 12.5)$ days, and the total postoperative hospital stay was $(26.3 \pm 20.5)$ days; see Table 3 .

\subsection{Follow-up results:}

The postoperative follow-up period was 3 62 $(30.3 \pm 15.5)$ months. During the follow-up period, 3 patients died, 1 patient died of septic shock after re-admission 2 months due to mediastinal infection, and 2 patients died of cerebral hemorrhage at 6 months and 18 months after operation. The specific cause of cerebral hemorrhage is unknown.

Table2. Etologies for reoperation [cases (\%)]

\begin{tabular}{|l|l|}
\hline Variable & Distribution \\
\hline $\begin{array}{l}\text { dilated aortic root/ascending aortic } \\
\text { aneurysm }\end{array}$ & $20(48.8)$ \\
\hline Aortic dissection & \\
\hline Debakey I & $3(7.3)$ \\
\hline Debakey II & $4(9.8)$ \\
\hline Pseudoaneurysm of aortic root & $4(9.8)$ \\
\hline Prosthetic valve endocarditis & $5(12.2)$ \\
\hline Paravalvular leak & $5(12.2)$ \\
\hline \multicolumn{2}{|r|}{ Note: Values are presented as $\mathrm{n}(\%)}$. \\
\hline
\end{tabular}

\section{Discussion:}

Aortic surgery has become more mature in my country. Aortic root replacement surgery or Bentall surgery has become a common surgery. Some large sample reports ${ }^{2-4}$ show that the risk of surgery has been greatly reduced. However, performing aortic root replacement surgery again, especially for patients who have had aortic root surgery before, is still a technically challenging operation $^{5-6}$. It is reported in the literature ${ }^{7}$ that the mortality rate of aortic root replacement has reached $18 \%$. To a large extent, this is closely related to the perioperative management of patients, the choice of reoperation methods and the previous surgical methods. Although the incidence of some serious complications requiring reoperation seems to have decreased, the number of patients undergoing reoperation due to various complications has increased. This may be related to the following points related. First, with the improvement of imaging technology, some patients with mild or no symptoms were detected early and received necessary surgical treatment. Second, the increase in the use of biological valves and the increase in valve-sparing operations have increased the reoperation rate. Third, as the age of patients undergoing heart surgery increases, reoperations due to postoperative aortic degeneration also increase. 
All heart surgery is dangerous when opening the chest, especially for patients undergoing re-open heart surgery. The mediastinum and pericardium are fixed due to postoperative adhesions, or huge aneurysms compress the sternum. We use a swing saw to open the sternum. At this time, important tissues may be damaged, and serious consequences may even occur.Dobell and Jain ${ }^{8}$ reported that the mortality rate of 30 patients with catastrophic hemorrhage during reopening of the aorta was $47 \%$. Therefore, we recommend that all patients undergoing re-cardiac surgery routinely undergo chest CT examination before surgery to assess the adhesion of the sternum to the aorta and the right heart, and routinely place surface defibrillation electrodes before surgery, so that ventricular fibrillation can be timely Perform defibrillation. Among the patients in this group, 25 patients $(61.0 \%)$ had femoral arteriovenous bypass before thoracotomy, and there were no complications such as ventricular fibrillation, cardiac arrest or heart injury during thoracotomy and separation of adhesions. This is basically consistent with the concept of Di Bartolomeo et al. ${ }^{9}$, that is, for patients with aortic aneurysms that are tightly connected to the sternum or whose distance is less than $10 \mathrm{~mm}, \mathrm{CPB}$ must be established before chest opening.

Table3. Operation details [cases $(\%) / \overline{\mathrm{x}} \pm \mathrm{s}]$

\begin{tabular}{|l|l|}
\hline Variable & Distribution \\
\hline Cardiopulmonary bypass & $168.1 \pm 41.2$ \\
\hline aortic block time & $84.5 \pm 22.3$ \\
\hline ICU stay time & $9.2 \pm 12.5$ \\
\hline Total postoperative hospital stay & $26.3 \pm 20.5$ \\
\hline Death during hospitalization & $6(14.6)$ \\
\hline Heart failure & $3(7.3)$ \\
\hline Septic shock & $3(7.3)$ \\
\hline Thoracotomy & $4(9.8)$ \\
\hline Postoperative complications & \\
\hline Stroke & $2(4.9)$ \\
\hline Tracheotomy & $3(7.3)$ \\
\hline Renal Failure & $5(12.2)$ \\
\hline Poor incision healing & $2(4.9)$ \\
\hline $\begin{array}{l}\text { Mechanical ventilation time }>\mathbf{7 2} \\
\text { h }\end{array}$ & $7(17.1)$ \\
\hline
\end{tabular}

Abbreviations: ICU, Intensive Care Unit.

Note: Values are presented as $\mathrm{n}(\%)$ or mean \pm standard deviation.
The main reason for this group of patients to undergo aortic root replacement after cardiac surgery was true aneurysm, followed by aortic dissection, pseudoaneurysm, PVE and paravalvular leak.Aortic valve disease combined with dilatation of the ascending aorta is a common clinical problem, because changes in stroke blood flow, aortic wall disease and mechanical Laplace law increase the risk of aortic rupture ${ }^{10-11}$.Aortic valve replacement alone can reduce the speed of aortic expansion, but it cannot reduce long-term adverse aortic events after surgery. In patients with preoperative expansion of the ascending aorta but not treated during the same period, the incidence of aortic adverse events during the 10 years was $14.28 \%^{12}$, mainly due to the increased risk of aortic dissection and aortic expansion and rupture.There is currently no gold standard for intervention in the diameter of the ascending aorta, but the overall trend is more positive. The intervention standard from $55 \mathrm{~mm}$ recommended by experts to $45 \mathrm{~mm}$ recommended by the guidelines indicates that long-term postoperative aortic events are receiving increasing attention ${ }^{13-14}$.In this group of data, 20 patients $(48.8 \%)$ underwent surgery again due to dilation of the aortic root and ascending aorta. Among them, 16 patients had preoperative aortic diameter $>45 \mathrm{~mm}, 2$ patients had aortic diameter $>40 \mathrm{~mm}$, and 2 patients of whom the aortic sinus aneurysm ruptured and penetrated into the pulmonary artery. Unfortunately, we did not collect data on the diameter of the aorta before the previous operation. We believe that a more detailed assessment before the previous operation and proper treatment of the dilated aorta at the same time should avoid reoperation of some similar patients.According to the literature ${ }^{15-16}$, the mortality rate of aortic disease secondary to aortic valve replacement, especially aortic dissection, is as high as $44 \%$. The recurring aortic Stanford Atype dissection is mainly ruptured at the junction of the ascending aorta and the sinus duct. Therefore, Bentall surgery can completely eliminate the rupture and reduce the risk of proximal rupture.In this group of data, 4 cases had ascending aortic dissection after previous aortic valve replacement, 1 case was located in the right coronary sinus, 1 case was sinus-tubular junction, and 2 cases were ascending aorta. One case of Debakey II dissection recurred after aortic dissection, and the breach was in the sinus. The occurrence of these dissections may have a certain relationship with the previous operation.According to literature ${ }^{17}$, in the long-term follow-up after Bentall surgery, the 
incidence of pseudoaneurysms was $8 \%$ to $15 \%$. In this group of data, 4 patients with pseudoaneurysm were all Bentall postoperative, 2 had coronary anastomotic tear, and 2 had valved catheter prosthesis and aortic root tear. In the previous operation, it is necessary to fully free the coronary artery opening to prepare buttons to ensure that the anastomosis is tension-free to avoid the occurrence of coronary anastomosis tears.Infective endocarditis is an important reason for reoperation after valve replacement, and it is also an important cause of death during hospitalization. In this group of data, 5 cases were re-operated due to PVE, including 3 cases of mechanical valve replacement surgery and 2 cases of biological valve replacement surgery. Among the 5 patients with paravalvular leakage, 4 were Behçet's disease. For this type of patients, before the previous operation, after fully assessing the condition, a more appropriate surgical plan can be selected to avoid reoperation.

In this group of data, a total of 6 patients died during postoperative hospitalization, 3 died of septic shock, and 3 died of heart failure. Among them, 3 patients had septic shock, 2 had mediastinal infection, and 1 had severe lung infection. Among the 2 patients with mediastinal infection, 1 had a recurrent aortic sinus dissection after a previous aortic dissection operation, which was difficult to stop bleeding, took a long time, had asthma attacks after removing the tracheal intubation, and had secondary debridement due to mediastinal infection, Repeated chills and fever, and finally received dialysis treatment due to renal failure; another case of Behcet's disease had paravalvular leakage and was treated with surgery. After surgery, he was given a large number of hormone shock treatments and then developed mediastinal infection.Early studies $^{18-19}$ reported that the mortality rate of mediastinal infection after cardiac surgery was as high as $20 \%$ to $50 \%$.In recent years, with the application of negative pressure sealing drainage technology, the treatment effect of mediastinal infection after cardiac surgery has been significantly improved, and the mortality rate and infection recurrence rate have decreased significantly, but the mortality rate is still about $5 \%$, and the infection recurrence rate has dropped to $10 \%$ or less ${ }^{20-21}$. Another patient with pulmonary infection was admitted to hospital with acute left heart failure and was assisted by tracheal intubation ventilator. After the operation, the tracheal intubation was removed and intubated a second time. Finally, the tracheotomy was performed. He breathed quickly and repeatedly fever. Eventually died of septic shock.Among the 3 patients who died of heart failure, 2 patients with aortic dissection involved the opening of the right coronary artery and had a history of coronary atherosclerotic heart disease. The coronary stent implantation was unsuccessful. The ventricular ejection fraction was $49 \%$, the CPB time was 133 minutes, the aortic occlusion time was 71 minutes, and the intraoperative minimum temperature was $32^{\circ} \mathrm{C}$. Another patient was admitted to the hospital for infective endocarditis, and his heart failure worsened and received emergency surgery. An annulus abscess was seen, and the abscess penetrated the posterior wall of the left ventricle. The CPB time was 196 minutes, the arterial occlusion time was 102 minutes, and the intraoperative minimum temperature was $30^{\circ} \mathrm{C}$. Considering that the main cause of early death of reoperation in the proximal aorta is heart failure ${ }^{2}$, appropriate intraoperative myocardial protection strategies and coronary perfusion are particularly important.In this group of patients, except for one case of retrograde perfusion through the coronary sinus, the rest were directly perfused through the coronary arteries. The perfusion effect was definite. Of the 2 patients who developed heart failure after surgery, one had heart failure caused by an abscess that penetrated the posterior wall of the left ventricle, and the other might be related to a history of coronary atherosclerotic heart disease.In re-aortic root replacement surgery, the replantation of the coronary arteries is often a key technical issue, especially in the case where a valved tube has been used to replace the aortic root, the coronary anastomosis must be carefully dissected to implant the new Graft. At the same time, the anastomosis between the transplanted valved tube and the coronary artery must be tension-free, and the coronary artery must be torsion-free to avoid bleeding or late rupture and pseudoaneurysms.In this group of cases, 2 patients underwent Bentall surgery before, and the coronary anastomosis was torn to form a pseudoaneurysm and they were treated again. One patient underwent great saphenous vein anastomosis to lengthen the right coronary artery due to difficulty in right coronary artery anastomosis. In 1 case, a bypass from the great saphenous vein to the proximal end of the right coronary artery was performed due to the narrow opening of the right coronary artery, and the opening of the right coronary artery was sewn and closed. We did not use the Cabrol technique 
because it may lead to the formation of blood clots in coronary artery grafts ${ }^{22}$.

Most surgeons tend to use mechanical valves for aortic root replacement surgery to avoid reoperation, but the risk of reoperation must be weighed against the risk of stroke and bleeding.In some studies ${ }^{23-24}$, $57 \%$ of patients underwent bioprosthetic valve replacement, and this part of patients was more free from thromboembolic complications after 10 years.In this group of data, 2 patients had stroke during hospitalization, and 2 patients died of cerebral hemorrhage during follow-up, which may be closely related to oral anticoagulants. In elderly patients, a new generation of biological valves may be safer, because biological valve failure is relatively rare in this age group ${ }^{25}$.

\section{Conclusions:}

In summary, the treatment of recurrent aortic root lesions after cardiac surgery is more difficult and the risk of surgery is higher. For each patient, a sufficient evaluation is required before surgery. Appropriate surgical approach, adequate myocardial protection, and a complete surgical plan are essential to ensure the success of the surgery.

Conflict of interest: all authors declare that there is no conflict of interest.

\section{References:}

[1] Bentall H, De Bono A. A technique for complete replacement of the ascending aorta. Thorax, 1968, 23(4): 338-339.

[2] Luciani N, De Geest R, Anselmi A, et al. Results of reoperation on the aortic root and the ascending aorta. Ann Thorac Surg, 2011,92(3):898-903.

[3] Jassar AS, Desai ND, Kobrin D, et al. Outcomes of aortic root replacement after previous aortic root replacement: The "true" redo root. Ann Thorac Surg, 2015, 99(5): 1601-1608.

[4] Etz CD, von Aspern K, Girrbach FF,et al. Long-term survival after composite mechanical aortic root replacement: a consecutive series of 448 cases.J Thorac Cardiovasc Surg. 2013, 145(3 Suppl):S417.

[5] Preventza O, Mohamed AS, Cooley DA, et al. Homograft use in reoperative aortic root and proximal aortic surgery for endocarditis:
A 12-year experience in high-risk patients. J Thorac Cardiovasc Surg. 2014,148(3):989994.

[6] Urbanski PP, Lakew F, Dinstak W, et al. Bentall procedure after previous aortic valve or complete root replacement: Usefulness of self-assembled aortic valve conduit. J Thorac Cardiovasc Surg, 2018, 156(1): 89-95.

[7] Shrestha M, Khaladj N, Baraki H, et al. Aortic root reoperation: A technical challenge. J Heart Valve Dis, 2010, 19(2): 177-181.

[8] Dobell AR, Jain AK. Catastrophic hemorrhage during redo sternotomy. Ann Thorac Surg, 1984, 37(4): 273-278.

[9] Di Bartolomeo R, Berretta P, Petridis FD, et al. Reoperative surgery on the thoracic aorta. J Thorac Cardiovasc Surg, 2013, 145(3 Suppl): S78-S84.

[10] Wu J, Zafar MA, Li Y, et al. Ascending Aortic Length and Risk of Aortic Adverse Events: The Neglected Dimension. J Am Coll Cardiol. 2019, 74(15):1883-1894.

[11] Davies RR, Goldstein LJ, Coady MA, et al. Yearly rupture or dissection rates for thoracic aortic aneurysms: Simple prediction based on size. Ann Thorac Surg, 2002, 73(1): 17-27.

[12] Matsuyama K, Usui A, Akita T, et al. Natural history of a dilated ascending aorta after aortic valve replacement. Circ J, 2005, 69(4): 392-396.

[13] Pitt MP, Bonser RS.The natural history of thoracic aortic aneurysm disease: an overview. J Card Surg. 1997,12(2 Suppl):270-278.

[14] Hiratzka LF, Bakris GL, Beckman JA, et al. 2010

ACCF/AHA/AATS/ACR/ASA/SCA/SCAI/ SIR/STS/SVM guidelines for the diagnosis and management of patients with thoracic aortic disease: Executive summary. A report of the American College of Cardiology Foundation/American Heart Association Task Force on Practice Guidelines, American Association for Thoracic Surgery, American College of Radiology, American Stroke Association, Society of Cardiovascular 
Anesthesiologists, Society for Cardiovascular Angiography and Interventions, Society of Interventional Radiology, Society of Thoracic Surgeons, and Society for Vascular Medicine. Catheter Cardiovasc Interv, 2010, 76(2): E43-E86.

[15] Tsutsumi K, Hashizume K, Inoue $\mathrm{Y}$. Natural history of the ascending aorta after aortic valve replacement: risk factor analysis for late aortic complications after aortic valve replacement.Gen Thorac Cardiovasc Surg. 2016, 64(5):243-50.

[16] von Kodolitsch Y, Loose R, Ostermeyer J, et al. Proximal aortic dissection late after aortic valve surgery: 119 cases of a distinct clinical entity. Thorac Cardiovasc Surg, 2000, 48(6): 342-346.

[17] Manenti A, Zizzo M, Fedeli C, et al. Coronary artery pseudoaneurysm after bentall procedure. Ann Thorac Surg, 2016, 102(4): 1409.

[18] Barbera F, Lorenzetti F, Marsili R, et al. The Impact of Preoperative NegativePressure Wound Therapy on Pectoralis Major Muscle Flap Reconstruction for Deep Sternal Wound Infections.Ann Plast Surg. 2019,83(2):195-200.

[19] Chello C, Lusini M, Nenna A, et al. Deep sternal wound infection (DSWI) and mediastinitis after cardiac surgery: current approaches and future trends in prevention and management. Surg Technol Int, 2020, 36: $212-216$.

[20] Fleck T, Fleck M. Negative pressure wound therapy for the treatment of sternal wound infections after cardiac surgery. Int Wound J, 2014, 11(3): 240-245.

[21] Lonie S, Hallam J, Yii M, et al. Changes in the management of deep sternal wound infections: a 12-year review. ANZ J Surg. 2015,85(11):878-881.

[22] Bachet J, Goudot B, Dreyfus G, et al. Current practice in Marfan's syndrome and annulo-aortic ectasia: Aortic root replacement with a composite graft over a twenty-year Period. J Card Surg, 1997, 12(2 Suppl): 157-166.
[23] Caynak B, Sagbas E, Onan B, et al. Comparison of three different surgical methods in aortic root aneurysms: longterm results. J Card Surg. 2009 NovDec;24(6):710-715.

[24] Sioris T, David TE, Ivanov J, et al. Clinical outcomes after separate and composite replacement of the aortic valve and ascending aorta. J Thorac Cardiovasc Surg, 2004, 128(2): 260-265.

[25] Masters RG, Haddad M, Pipe AL, et al. Clinical outcomes with the Hancock II bioprosthetic valve.Ann Thorac Surg. 2004,78(3):832-836. 